\title{
Depression in people with type 2 diabetes: current perspectives
}

This article was published in the following Dove Press journal: Diabetes, Metabolic Syndrome and Obesity:Targets and Therapy

\author{
Lina Darwish ${ }^{1-4}$ \\ Erika Beroncal ${ }^{1-3}$ \\ Ma Veronica Sison ${ }^{1-3}$ \\ Walter Swardfager ${ }^{1-4}$
}

'Hurvitz Brain Sciences Program, Sunnybrook Research Institute, Toronto, ON, Canada; ${ }^{2}$ Department of Pharmacology and Toxicology, University of Toronto, Toronto, ON, Canada; ${ }^{3}$ Cardiac Rehabilitation Program, University Health Network Toronto Rehabilitation Institute, Toronto, ON, Canada; ${ }^{4}$ Canadian Partnership for Stroke Recovery, Toronto, ON, Canada
Correspondence: Walter Swardfager Hurvitz Brain Sciences Program, Sunnybrook Research Institute, 2075 Bayview Avenue, Toronto, ON M4N $3 \mathrm{M} 5$, Canada

Tel +l 4164806100

Email w.swardfager@utoronto.ca

\begin{abstract}
Type 2 diabetes mellitus (T2DM) is associated with depressive symptoms, and comorbid depression in those with T2DM has been associated with adverse clinical profiles. Recognizing and addressing psychological symptoms remain significant clinical challenges in T2DM. Possible mediators of the reciprocal relationship between T2DM and depression may include physical activity levels, effectiveness of self-management, distress associated with a new T2DM diagnosis, and frailty associated with advanced diabetes duration. The latter considerations contribute to a "J-shaped" trajectory from the time of diagnosis. There remain significant challenges to screening for clinical risks associated with psychological symptoms in T2DM; poorer outcomes may be associated with major depressive episodes, isolated (eg, anhedonic), or subsyndromal depressive symptoms, depressive-like symptoms more specific to T2DM (eg, diabetes-related distress), apathy or fatigue. In this review, we discuss current perspectives on depression in the context of T2DM with implications for screening and management of these highly comorbid conditions.
\end{abstract}

Keywords: type 2 diabetes, depression, mood, complications

\section{Introduction}

The likelihood of depression in type 2 diabetes mellitus (T2DM) is approximately double that found in the general population. ${ }^{1-3}$ The cardinal symptoms of a major depressive episode according to Diagnostic And Statistical Manual Of Mental Disorders, Fifth Edition criteria are sadness and/or anhedonia with additional symptoms of decreased energy, changes in thinking, appetite changes, disrupted sleep, or suicidality. ${ }^{4,5}$ These symptoms may occur together or in isolation in people with T2DM. Depression or depressive symptoms have been associated with adverse clinical profiles, including poorer glycemic control, eating habits, ${ }^{6,7}$ and exercise adherence ${ }^{8}$ in those with T2DM. Despite their importance, recognizing and addressing psychological symptoms in T2DM remain significant clinical challenges.

There has been considerable debate over the past decade about the most important symptom clusters, psychological constructs, and screening tools. Clinical outcomes have been associated with specific symptoms of major depression (eg, anhedonia), ${ }^{9,10}$ isolated or subsyndromal depressive symptoms that are not part of a depressive episode, ${ }^{7}$ fatigue, or depressive-like symptoms more specific to the burden of T2DM (ie, diabetes-related distress). ${ }^{2,9,11}$ Recent studies suggest that duration of diabetes may be an important factor in the temporal trend of depressive symptoms at the population level, likely due to the development and severity of diabetes-related distress and 
frailty. ${ }^{12-15}$ In this review, we discuss current perspectives on diabetes and depression with implications for screening and management of these highly comorbid conditions. Our primary aims were to summarize temporal trends in depressive symptoms, overlapping psychosocial constructs and the instruments used to assess them, possible implications for pharmacotherapy, and the impact of comorbid diabetes and depression on longer-term outcomes.

\section{Depression and diabetes: a reciprocal causal relationship?}

Questions remain concerning causality in the reciprocal relationships identified between diabetes and depression. ${ }^{15,16}$ Epidemiological studies indicate that depression is a risk factor for future diabetes ${ }^{17,18}$ and that diabetes is a risk factor for future depression. ${ }^{19}$ It has been proposed that depressive symptoms act as mediators of subsequent metabolic disruptions due to their effects on activity levels and other health behaviors..$^{20,21}$

Some evidence that T2DM can cause depression has been gleaned from Mendelian randomization studies, ${ }^{22-24}$ in which single-nucleotide polymorphisms known to predispose T2DM were also predictors of anhedonic, interpersonal, somatic, and other depressive symptoms - suggesting that diabetes can cause depressive symptoms. ${ }^{25}$ It has been suggested that distress associated with a new T2DM diagnosis might precipitate or exacerbate depressive symptoms ${ }^{19}$ as in other newly diagnosed chronic diseases. ${ }^{16,26}$ That view would be consistent with elevated depressive symptoms in patients diagnosed clinically with diabetes compared to those with undiagnosed diabetes. ${ }^{27}$ Both a diagnosis of diabetes ${ }^{19}$ and treatment for diabetes ${ }^{16,28,29}$ have been associated with an increased likelihood of depression.

\section{Depression, distress, and other discontent in T2DM}

About $18 \%-25 \%$ of people with T2DM will meet DSM criteria for a major depressive episode using the Structured Clinical Interview for DSM-5 Axis-I Disorders (SCID), a prevalence at least double that is found in the general population. ${ }^{30-32}$ Most clinicians would agree that when identified, treatment should be offered for major depressive disorder (MDD); however, depression is a heterogeneous condition, ${ }^{33}$ and not all symptoms of MDD are related to diabetes outcomes in the same way. Of particular interest, anhedonia can occur as a cardinal symptom of a major depressive episode, as an isolated symptom, as a personality trait, or as a neuroendocrine symptom of T2DM. ${ }^{9}$ According to the DSM-5, anhedonia is characterized by the "lack of enjoyment from, engagement in, and energy for life experiences". ${ }^{34}$ Anhedonia has been associated with suboptimal glucose control, increasing the odds of having HbAlc levels $\geq 7$ by $30 \%{ }^{10}$ Moreover, in a prospective study, anhedonia specifically (not dysphoria) increased the risk of mortality, and this was mediated by physical activity. ${ }^{35}$ Given that this cardinal depressive symptom can occur in patients with T2DM, in constellation with other depressive symptoms that might meet criteria for a diagnosis of MDD, it might be useful to consider that some cases might be more reflective of a diabetes-related depressive syndrome rather than MDD per se. Basic neuroscience studies suggest different neuroanatomical and neurochemical underpinnings of different facets of anhedonia (eg, consummatory, decisional, motivational, and anticipatory), ${ }^{36}$ which are not generally discerned in structured clinical interviews. Motivational and/or decisional aspects of anhedonia may link dopaminergic dysfunction with diabetes-related distress, major depression, and fatigue, which might interfere with the management of diabetes. ${ }^{9}$

Depression heightens the psychological impact of a diagnosis of diabetes, resulting in increased diabetes-related distress. ${ }^{37}$ Diabetes-related distress is a construct more specific to the psychological burden of a diagnosis of diabetes and its management, ${ }^{2,38}$ and many of its tenets overlap with the symptoms of major depression. ${ }^{39,40}$ As shown in Figure 1, the constructs of depression and distress capture elements of fatigue, ${ }^{4,41}$ anhedonia, and dysphoria. ${ }^{2,42}$ These constellations of symptoms may result in functional impairment, problems in self-management, poorer glycemic control, increased risk of diabetes complications, and poorer quality of life. ${ }^{2}$

One specific domain of diabetes-related distress relates to the burden of management. Patients prescribed a multifactorial treatment plan show more distress in their first year following diagnosis, but patients undergoing less intensive treatments may not develop distress for several years after diagnosis. ${ }^{43}$ As the burden of management increases, so do symptoms of distress; in a Chinese population-based study, burden of insulin use, but not depression, correlated with the duration of diabetes. ${ }^{44}$ Conceivably, the need to use insulin can lead to negative health perceptions, resulting in increased distress. Accordingly, in another study, diabetes-related distress, but not depression, correlated with HbA1c. ${ }^{45}$ This might be expected since unlike depression, diabetes-related distress is defined by specific, contextual stressors relating to diabetes, also indexing the impact of depression. ${ }^{38}$

Fatigue is associated with poorer day-to-day functioning attributable to symptoms of tiredness, lack of energy, 


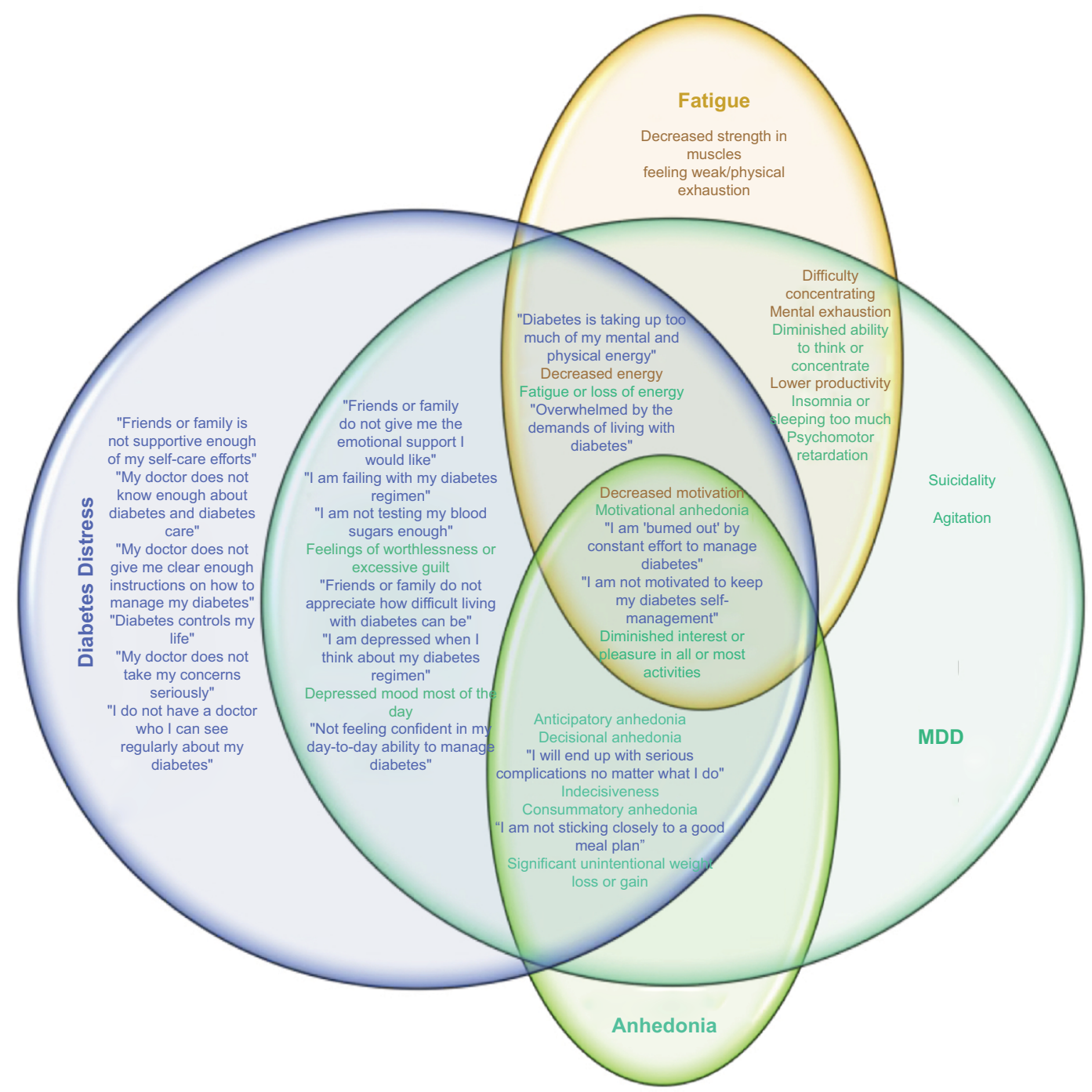

Figure I Venn diagram exploring intersections between symptoms of MDD (teal), diabetes-related distress (blue), fatigue (orange), and anhedonia (green).

Notes: Items are derived from the DDS- $17^{133}$ and Diagnostic And Statistical Manual Of Mental Disorders-5 diagnostic criteria for a depressive episode. ${ }^{5}$ Both diabetes-related distress and depression can share symptoms of fatigue ${ }^{41,46,47}$ and/or anhedonia (including anticipatory, motivational, decisional, and consummatory dimensions).

Abbreviations: DDS, Diabetes Distress Scale; MDD, major depressive disorder.

or exhaustion. ${ }^{41}$ It is differentiated from an acute state of tiredness (which can occur normally) as a chronic state whereby the body is nonresponsive to rest. Fatigue is a barrier to participation in physical activities and to engagement in self-care activities in T2DM. ${ }^{41}$ Both DSM depression criteria and many screening tools for depressive symptoms, such as the Center for Epidemiologic Studies Depression Scale (CES-D), include symptoms of fatigue. Notably, in a recent validation of the CES-D in T2DM, the fatigue item was found, in differential item functioning testing, to be non-invariant with respect to glycemic control, suggesting that fatigue in T2DM was more closely related to symptoms of T2DM than symptoms of depression per se. ${ }^{46}$ This might inflate depressive symptom estimates, particularly "somatic symptoms" in
T2DM, and affect the accuracy of screening. Similarly, the probability to endorse the item "feeling that diabetes is taking up too much of my mental and physical energy every day" on the Diabetes Distress Scale (DDS ${ }^{47}$ is likely to be related to fatigue and also to an inability to apply self-care strategies. In patients with $\mathrm{T} 2 \mathrm{DM}$ with an $\mathrm{HbA} 1 \mathrm{c}>7 \%$, fatigue is associated with pain and inflammation. ${ }^{47}$ Anhedonia and fatigue have been related to inflammation ${ }^{48}$ and they may be convergent constructs in T2DM. One study, aiming to understand complications associated with newly diagnosed patients with T2DM, found that fatigue was reported by $61 \%$ of patients. In that study, fatigue was significantly associated with fasting plasma glucose but not with HbAlc. ${ }^{49}$ Another construct closely related to anhedonia and fatigue is apathy, 
which can be screened using the Apathy Evaluation Scale or diagnosed based on a clinical assessment. ${ }^{50}$ Symptoms of apathy are more common in T2DM, and they are related to depression, poorer glycemic control, and cognitive decline. ${ }^{51}$

\section{Duration of diabetes and depression}

For many of the reasons mentioned earlier, duration of diabetes influences depressive symptoms, contributing to a "J-shaped" curve over time in T2DM as shown in Figure 2 . In general, depressive symptoms elevate immediately following diagnosis and then decrease over several years, increasing again with longer duration. In one study using a cutoff of 7 on the Geriatric Depression Scale (GDS), durations of diabetes $<10$ years or $>30$ years were associated with increased odds of depression, whereas durations such as $10-30$ years were not. ${ }^{12}$ The increase in depression with longer durations of diabetes was shown to be mediated by increased frailty scores. ${ }^{12}$

In another T2DM population, about $40 \%$ of the study population was depressed according to the Patient Health Questionnaire (PHQ)-9 with shorter duration of diabetes predicting increased occurrence of depression. ${ }^{13}$ However, in a study examining the relationship between various chronic conditions and depressive symptoms, no increase in depressive symptoms was observed after initial diagnosis of diabetes $;{ }^{14}$ instead, specific to diabetes, there was a gradual increase in these symptoms over time, consistent with the latter portion of the J-shaped model. In another study, a peak in depressive symptoms, as measured by the CES-D, was observed at baseline, with a gradual decrease over the next 6 years, also consistent with the proposed J-shaped model of depressive symptoms in T2DM. ${ }^{15}$ Although not specifically tested, these, the initial increase in depressive symptoms in shorter durations, might be related to distress associated with a diagnosis and newly imposed management regimes (eg, additional medications, checking blood sugar, dieting, and exercise). ${ }^{49}$ An increased incidence of depression has been observed within the first year of initiation of an oral antidiabetic medication. ${ }^{50}$ After an adjustment to management and/or remaining generally asymptomatic with respect to diabetes complications, depressive symptoms may then subside. Some studies have suggested that older adults with T2DM have been more successful in meeting T2DM outcome standards when compared to younger adults, and this was not due to either increased disease knowledge or shorter disease duration. ${ }^{53-55}$ In these studies, the duration of diabetes of the older patients falls within the dip in our proposed J-shaped curve, supporting the suggestion that this relatively longer duration of T2DM may be associated with adjustment to diabetes-related lifestyle changes. With further increased

Duration of diabetes and prevalence of depressive symptoms

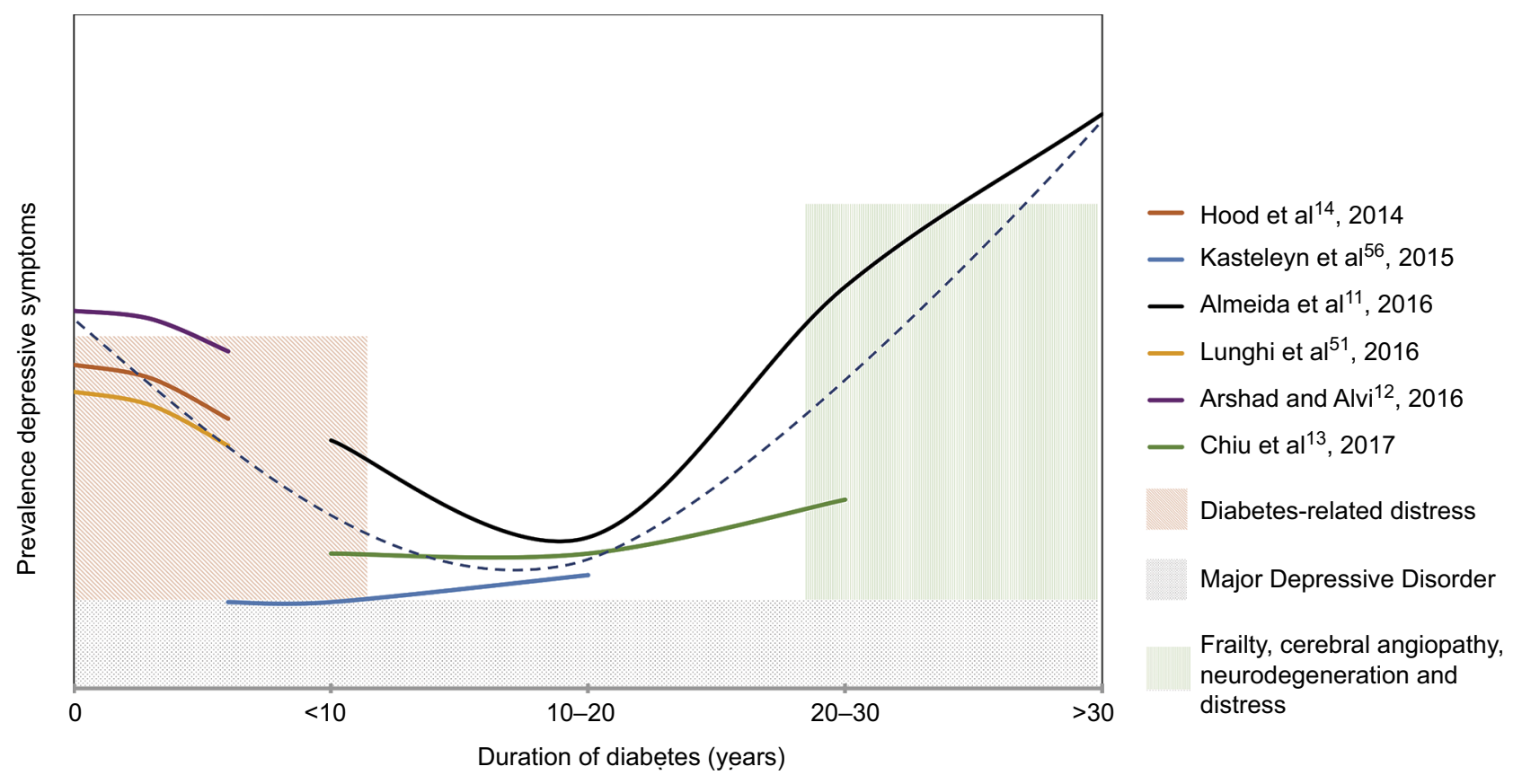

Figure 2 Temporal trend in depressive symptoms with duration of diabetes.

Notes: Results from separate studies reporting trends in depressive symptoms (colored lines) suggest a J-shaped curve (dashed line). Initial and later peaks are observed, which are thought to result from different biopsychosocial factors (colored shaded bars). The high prevalence of MDD (I8\%-25\%) contributes to the overall prevalence of depressive symptoms, but temporal trends with duration of diabetes have not been described explicitly.

Abbreviation: MDD, major depressive disorder. 
duration of diabetes, cerebral microvascular complications may contribute to symptoms such as anhedonia and apathy. ${ }^{56}$ Both macrovascular and microvascular complications have been associated with an increase in incident depressive symptoms. ${ }^{57}$ In addition, more taxing management regimes may increase distress; ${ }^{58}$ for instance, the need for insulin injection may lead to a negative outlook on overall health. ${ }^{59-61}$ Moreover, at longer durations of diabetes, other chronic comorbidities typically accumulate, resulting in increased frailty, which is characterized by decreased physical capability, increased exhaustion, and poorer weight loss outcomes ${ }^{62,63}$ in T2DM. Because depression is a heterogeneous condition, more data on temporal trends in individual depressive symptom domains will be important to discern in future studies.

\section{Depression screening and diagnosis}

Depression is diagnosed in a structured clinical interview, such as the SCID. ${ }^{64,65}$ Other interviews include the following: the Montgomery-Asberg Depression Rating Scale (MADRS) and the Composite International Diagnostic Interview (CIDI). However, these interviews vary in length and they can be time-consuming and increasing patient burden, and depression can be screened accurately using as few as two questions (eg, the PHQ-2).

The high rate of comorbid depression in T2DM, and the heterogeneity of its symptoms, necessitates some additional considerations in this population. The accuracy of the tests can vary between populations, necessitating comparison against the gold standard interviews to determine optimal cutoff scores to detect depression. In addition, psychometric evaluation is required to determine the suitability of the individual items and overall construct validity specifically in T2DM. ${ }^{66}$ The properties of various screening instruments and their validities in T2DM remain an ongoing area of study. ${ }^{67}$ Differential item functioning is one analysis that can be used to determine whether the questions on each are non-invariant with respect to T2DM and/or T2DM characteristics to ensure that they accurately measure depressive symptoms rather than symptoms of T2DM. ${ }^{68,69}$ Self-report screening questionnaires used in T2DM include the following: the CES-D, ${ }^{46}$ Beck Depression Inventory (BDI), ${ }^{70}$ World Health Organization (WHO) Well-Being Index, ${ }^{71} \mathrm{PHQ},{ }^{72}$ and Edinburg Depression Scale (EDS), among others. ${ }^{67}$ The self-report scales used most commonly in T2DM, and studies assessing their validity, have been reviewed recently. ${ }^{67}$

One of the most commonly used and best supported questionnaires to screen for depression is the CES-D, ${ }^{67}$ which contains 20 items and is used to assess depressive symptoms that occur in the previous week with individual item scores ranging from 0 to 3 . Scores can range from 0 to 60 with higher scores indicating the presence of more depressive symptoms, and a cutoff of 16 or greater is suggested to indicate significant depressive symptoms in the general population. Although this cutoff was appropriate to predict non-completion of exercise in patients with coronary artery disease, ${ }^{73}$ a cutoff of 10 was found to predict non-completion of exercise in T2DM. ${ }^{7}$ An abridged (14-item) version of the CES-D was proposed based on invariance testing, ${ }^{74}$ and the 14-item version was validated for use in patients with T2DM, ${ }^{46}$ although optimal cutoffs to detect a major depressive episode, and risks of non-adherent behaviors, remain to be determined. The subdomains capture anhedonia, sadness, and somatic symptoms, the latter two almost perfectly correlated, and all of the items are best viewed as a single measure of depression overall. ${ }^{46}$

The Beck Depression Inventory (BDI) was originally designed to capture the severity of depression using 21 items indicating psychological and somatic symptoms in the previous week with four ordered response categories. The cutoff for the general population using the BDI-I is a summed score of 13 or greater, but a cutoff of 16 or greater was suggested to be more accurate in diabetes. ${ }^{70,75}$ Using this cutoff, there is an overall prevalence of $32.8 \%$ in diabetes. ${ }^{76}$ The BDI has been revised (BDI-II), although the psychometric properties of the scale and possible subdomains remain to be explored in-depth in T2DM. ${ }^{77-79}$

The WHO Well-Being Index is unique in that it contains five positively worded items that assess the absence of positive mood during the previous 2 weeks. It utilizes a 6-point frequency scale resulting in a well-being score that can range from 0 to $25{ }^{80,81} \mathrm{~A}$ higher well-being score is considered to be an overall indicator of positive mental health, but items do not quantify any specific subdomains of depression. ${ }^{82}$ The cutoff for the general population that indicates "poor" well-being is 13 or lower, but the ideal cutoff for patients with T2DM was found to be 10 or lower. ${ }^{80}$ Approximately $79.5 \%$ of respondents with T2DM were correctly described to have an absence of depressive symptoms based on this cutoff score. ${ }^{80,81}$

Another effective screening tool is Patient Health Questionnaire (PHQ)-9, which contains nine items that measure the frequency of depressive symptoms with a 4-point Likert scale. In this review, the scale items capture anhedonia, depressed mood, insomnia, and energy level. A total score of 10 or greater is indicative of depression in the general population, but for patients with T2DM, a cutoff of 12 or greater was found to be more effective. ${ }^{72}$ It has been suggested that just two questions (the PHQ-2), which probe the 
two cardinal symptoms of MDD, can also detect MDD with high accuracy in other populations. ${ }^{83}$

The EDS is a unidimensional questionnaire consisting of 10 items with a 4-point rating scale. The presence of major depression is determined by a score of 12 or 13 , whereas scores ranging from 9 to 11 indicate the presence of mild depression. An advantage of using this as a screening tool for depression is the absence of bias by somatic symptoms caused by diabetes itself. Because this test was originally developed to measure postnatal depression, bias by gender-related symptoms may be a concern when summing the scores. ${ }^{81,84}$ Using the EDS, $9.8 \%$ of men with diabetes showed symptoms of mild depression and $8.1 \%$ showed severe depressive symptoms, while $16.5 \%$ of females showed symptoms of mild depression and $16.2 \%$ showed symptoms of severe depression. ${ }^{84}$ While some have reported that the EDS is multidimensional (quantifying anhedonia, anxiety, and depression), ${ }^{85,86}$ confirmatory factor analysis has shown that EDS is unidimensional and measures a general depression factor. ${ }^{84}$

\section{Interventions for comorbid depression and diabetes}

Studies suggest that the treatment of comorbid depression and T2DM is more effective when embedded in an integrated approach, in which both conditions are addressed together. ${ }^{87}$ Pharmacological treatment with antidepressants such as selective serotonin reuptake inhibitors (SSRIs) have been shown to be effective ${ }^{88}$ and to prevent the recurrence of depression in patients with diabetes. ${ }^{89}$ Bupropion, a norepinephrine and dopamine (DA) reuptake inhibitor, would seem to be at least equally effective as SSRIs in T2DM; ${ }^{90}$ however, it has not been compared head to head with an SSRI in a randomized trial. Treatment with SSRIs or bupropion can help patients achieve better glycemic control. ${ }^{90}$ No antidepressant therapy has been specifically indicated based on a comorbid diagnosis of T2DM nor have been any particular antidiabetic regimens that might have varying effects on mood indicated for comorbid depression in the setting of T2DM. For instance, in animal models, the glucagon-like peptide 1 agents appear to have antidepressant properties, ${ }^{91}$ warranting further studies in human beings.

DA plays an important role in both depression and T2DM. There is evidence that a decrease in DA signaling in the striatum occurs in T2DM, and in rodent models of obesity, which could result in decreased psychomotor activity, motivation, and dysfunction of reward systems..$^{92}$ Apart from its antidepressant properties, DA enhances glycemic control and insulin sensitivity. ${ }^{93}$ DA agonists (ie, bromocriptine) can decrease food intake and increase locomotor activity. ${ }^{94}$ Another dopaminergic agent, methylphenidate, has been shown to be effective against symptoms of apathy. ${ }^{95,96}$ Therefore, agents that increase DA may be more helpful in the management of depression in the context of T2DM compared to other pharmacological agents, as they may improve apathy, depressive symptoms, and glycemic control, and they may also assist with cognition and motivation to improve lifestyle factors.

Anti-inflammatory agents are under investigation for depression in the setting of inflammatory comorbidity and treatment-resistant depression. A bidirectional relationship between inflammation and T2DM has been observed, and depression is associated with inflammation in T2DM, ${ }^{97,98}$ suggesting that anti-cytokine ${ }^{99,100}$ or other anti-inflamamtory ${ }^{101,102}$ approaches may be useful as additional or adjunctive therapies.

Non-pharmacological interventions, including cognitive behavioral therapy (CBT), ${ }^{103}$ interpersonal therapy, ${ }^{104}$ and exercise intervention programs, ${ }^{8,105,106}$ can be prescribed. CBT utilizes behavioral strategies, problem-solving procedures, and cognitive techniques in treatment. ${ }^{103}$ Alongside diabetes education, CBT was found to be effective, with $58.3 \%$ of patients in the CBT group achieving remission vs $25.9 \%$ who received diabetes education alone. ${ }^{103}$ In that study, glycemic control was improved; however, in a more recent study, CBT showed no benefit on glycemic control and less effectiveness than sertraline on glycemic control. ${ }^{107}$

Interpersonal therapy is a personalized approach that recognizes cultural, social, and psychological contexts that may affect adherence to medication or treatment. ${ }^{104}$ Discussion of treatment options and education can be integrated into this framework to optimize medication use. ${ }^{108}$ In one study, the intervention of an integrated care manager had significant effects on clinical outcomes for both depression and diabetes, which was due to increased adherence to medications and treatment recommendation. ${ }^{104}$

Exercise improves depressive symptoms, ${ }^{106,109}$ glucose disposal, insulin sensitivity, and glycemic control. ${ }^{105,110}$ This may be partially attributed to recruitment of DA and striatal circuits for reward and stress resistance. ${ }^{111}$ However, a systematic review highlighted heterogeneity in the effects of exercise on psychological outcomes in T2DM. ${ }^{112}$ Although many benefits of exercise appear to occur regardless of exercise modality, ${ }^{105,110}$ that work suggests the need to identify optimal regimes and/or predictors of response.

Multiple approaches were utilized in a three-phase collaborative primary team model designed for patients 
with comorbid diabetes and depression. The three phases included the following: improvement in depressive symptoms; improvement in blood glucose, blood pressure, and cholesterol; and improvement in self-care behaviors. This collaborative care model was found to have benefit in both diabetes and depression outcomes, emphasizing the benefit of a holistic approach. ${ }^{113}$

\section{Clinical sequelae of depression in diabetes}

As discussed, depression in T2DM has been associated with poorer glycemic control in some, ${ }^{6,30}$ but not all ${ }^{13,114}$ studies. Moreover, treatment for depression improves glycemic control in some, ${ }^{90,93,103,110}$ but not all ${ }^{115,116}$ studies. One study investigating the effect of bupropion on depressed patients with T2DM found mood-related reductions in HbA1c, independent of changes in diabetes self-care practices. ${ }^{117}$ Some discrepancies between studies may have to do with the symptoms assessed or heterogeneity in depression diagnoses. For instance, anhedonic symptoms may correlate more closely with glycemic control than other depressive symptoms $s^{9,10,35,118,119}$ and with prospective mortality risk due to their effects on physical activity. ${ }^{35}$ Depression in people with diabetes increases the risks of stroke, cardiovascular mortality, ${ }^{120}$ and all-cause mortality. ${ }^{121-123}$

Both T2DM and depression are associated with a decline in cognitive function due to multiple effects on the brain. For instance, diabetes is associated with an increased rate of cortical atrophy, ${ }^{124}$ microvascular brain disease, and deficits in cerebral blood flow. ${ }^{125,126}$ A population-based study found that having the two conditions together was associated with a more-than-additive effect in increasing the risk of dementia. ${ }^{127}$ Diabetes and depression co-occurring in a post-stroke population also had a cumulative impact on executive function and on the risk of severe vascular cognitive impairment. ${ }^{128}$ It is conceivable that diabetes and depression co-contribute a neural environment that places the brain at risk for dementia due to inflammation, ${ }^{98}$ neuroendocrine changes associated with chronic stress, ${ }^{129}$ microangiopathic changes, and an increased propensity for subcortical infarcts. ${ }^{130}$ Notably, educational attainment has been found to decrease the risk of depression in people with T2DM, ${ }^{13,131}$ which might bolster cognitive reserve against brain loss. Sophisticated mediation modeling has been used to examine relationships between diabetes, depression, and cognitive outcomes over time, demonstrating a direct effect of T2DM on cognition, and an indirect effect that is mediated by depression. ${ }^{132}$ Studies of this type might also be useful to disentangle the temporal relationships between diabetes and depression themselves.

\section{Conclusion}

Although there is debate surrounding the bidirectional relationship between diabetes and depression, it is clear that the two conditions occurring together can make both conditions more difficult to manage, and contribute additively to adverse long-term sequelae such as mortality, stroke, and dementia. There are challenges in identifying the most harmful symptoms of depression in the context of diabetes with subsyndromal symptoms and depressionlike symptoms posing considerable barriers to effective management. Due to overlap in the multiple relevant psychological symptoms, it will be important to examine further the properties of different psychometrics, including those designed to capture more specific subdomains, in T2DM, with careful attention to how these constructs may uniquely predict different aspects of the management of diabetes and clinical outcomes over the different epochs of duration of diabetes. It may be particularly important to recognize and distinguish between diabetes-related distress, frailty, fatigue, apathy, anhedonia, and clinical depression in studies assessing the effectiveness of both pharmacological and non-pharmacological treatment modalities for T2DM. Particular attention should be paid to manage T2DM and psychological conditions together.

\section{Disclosure}

The authors report no conflicts of interest in this work.

\section{References}

1. Mommersteeg PMC, Herr R, Pouwer F, Holt RIG, Loerbroks A. The association between diabetes and an episode of depressive symptoms in the 2002 World Health Survey: an analysis of 231797 individuals from 47 countries. Diabet Med. 2013;30(6):e208-e214.

2. Fisher L, Skaff MM, Mullan JT, et al. Clinical depression versus distress among patients with type 2 diabetes: not just a question of semantics. Diabetes Care. 2007;30(3):542-548.

3. Nouwen A, Winkley K, Twisk J, et al. Type 2 diabetes mellitus as a risk factor for the onset of depression: a systematic review and metaanalysis. Diabetologia. 2010;53(12):2480-2486.

4. Lopez Molina MA, Jansen K, Drews C, Pinheiro R, Silva R, Souza L. Major depressive disorder symptoms in male and female young adults. Psychol Health Med. 2014;19(2):136-145.

5. American Psychiatric Association. Diagnostic and statistical manual of mental disorders. 5th ed. Arlington, VA: American Psychiatric Publishing; 2013.

6. Lin EHB, Katon W, Von Korff M, et al. Relationship of depression and diabetes self-care, medication adherence, and preventive care. Diabetes Care. 2004;27(9):2154-2160.

7. Gonzalez JS, Peyrot M, McCarl LA, et al. Depression and diabetes treatment nonadherence: a meta-analysis. Diabetes Care. 2008;31(12):2398-2403. 
8. Swardfager W, Yang P, Herrmann N, et al. Depressive symptoms predict non-completion of a structured exercise intervention for people with type 2 diabetes. Diabet Med. 2016;33(4):529-536.

9. Carter J, Swardfager W. Mood and metabolism: anhedonia as a clinical target in type 2 diabetes. Psychoneuroendocrinology. 2016;69:123-132.

10. Nefs G, Pouwer F, Denollet J, Kramer H, Wijnands-van Gent CJ, Pop VJ. Suboptimal glycemic control in type 2 diabetes: a key role for anhedonia? J Psychiatr Res. 2012;46(4):549-554.

11. Naicker K, Øverland S, Johnson JA, et al. Symptoms of anxiety and depression in type 2 diabetes: associations with clinical diabetes measures and self-management outcomes in the Norwegian HUNT study. Psychoneuroendocrinology. 2017;84:116-123.

12. Almeida OP, McCaul K, Hankey GJ, et al. Duration of diabetes and its association with depression in later life: the Health in Men Study (HIMS). Maturitas. 2016;86:3-9.

13. Arshad AR, Alvi KY. Frequency of depression in type 2 diabetes mellitus and an analysis of predictive factors. J Pak Med Assoc. 2016;66(4):425-429.

14. Chiu CJ, Hsu YC, Tseng SP. Psychological prognosis after newly diagnosed chronic conditions: socio-demographic and clinical correlates. Int Psychogeriatr. 2017;29(2):281-292.

15. Hood KK, Beavers DP, Yi-Frazier J, et al. Psychosocial burden and glycemic control during the first 6 years of diabetes: results from the SEARCH for diabetes in youth study. J Adolesc Health. 2014;55(4):498-504.

16. Tabák AG, Akbaraly TN, Batty GD, Kivimäki M. Depression and type 2 diabetes: a causal association? Lancet Diabetes Endocrinol. 2014;2(3):236-2452.

17. Rotella F, Mannucci E. Depression as a risk factor for diabetes: a metaanalysis of longitudinal studies. J Clin Psychiatry. 2013;74(1):31-37.

18. Eaton WW, Armenian H, Gallo J, Pratt L, Ford DE. Depression and risk for onset of type II diabetes: a prospective population-based study. Diabetes Care. 1996;19(10):1097-1102.

19. Rotella F, Mannucci E. Diabetes mellitus as a risk factor for depression. A meta-analysis of longitudinal studies. Diabetes Res Clin Pract. 2013;99(2):98-104.

20. Moulton CD, Pickup JC, Ismail K. The link between depression and diabetes: the search for shared mechanisms. Lancet Diabetes Endocrinol. 2015;3(6):461-471

21. Renn BN, Feliciano L, Segal DL. The bidirectional relationship of depression and diabetes: a systematic review. Clin Psychol Rev. 2011;31(8):1239-1246.

22. Lawlor DA, Harbord RM, Tybjaerg-Hansen A, et al. Using genetic loci to understand the relationship between adiposity and psychological distress: a Mendelian Randomization study in the Copenhagen General Population Study of 53221 adults. J Intern Med. 2011;269(5):525-537.

23. Hung CF, Rivera M, Craddock N, et al. Relationship between obesity and the risk of clinically significant depression: Mendelian randomisation study. Br J Psychiatry. 2014;205(1):24-28.

24. Hartwig FP, Bowden J, Loret De Mola C, Tovo-Rodrigues L, Davey Smith G, Horta BL. Body mass index and psychiatric disorders: a Mendelian randomization study. Sci Rep. 2016;6:32730.

25. Brewster P, Gross A, Gibbons LE, Mukherjee S, Crane PK, Glymour MM. The structure of depression in relation to type II diabetes: a Mendelian randomization analysis. Gerontologist. 2015;55:525-525.

26. Almeida OP. Vascular depression: myth or reality? Int Psychogeriatrics. 2008;20(4):645-652.

27. Nouwen A, Nefs G, Caramlau I, et al. Prevalence of depression in individuals with impaired glucose metabolism or undiagnosed diabetes: a systematic review and meta-analysis of the European Depression in Diabetes (EDID) research consortium. Diabetes Care. 2011;34(3):752-762.

28. Kivimki M, Batty GD, Jokela M, et al. Antidepressant medication use and risk of hyperglycemia and diabetes mellitus-a noncausal association? Biol Psychiatry. 2011;70(10):978-984.

29. Wahlqvist ML, Lee MS, Chuang SY, et al. Increased risk of affective disorders in type 2 diabetes is minimized by sulfonylurea and metformin combination: a population-based cohort study. BMC Med. 2012;10:150.
30. Papelbaum M, Moreira RO, Coutinho W, et al. Depression, glycemic control and type 2 diabetes. Diabetol Metab Syndr. 2011;3(1):26.

31. De Groot M, Jacobson AM, Samson JA, Welch G. Glycemic control and major depression in patients with type 1 and type 2 diabetes mellitus. J Psychosom Res. 1999;46(5):425-435.

32. Pibernik-Okanovic M, Peros K, Szabo S, Begic D, Metelko Z. Depression in Croatian type 2 diabetic patients: prevalence and risk factors. A Croatian survey from the European Depression in Diabetes (EDID) Research Consortium. Diabet Med. 2005;22(7):942-945.

33. Goldberg D. The heterogeneity of "major depression.". World Psychiatry. 2011;10(3):226-228.

34. American Psychiatric Association. DSM- $V$. Washington, DC: APA; 2013.

35. Nefs G, Pop VJM, Denollet J, Pouwer F. Depressive symptoms and all-cause mortality in people with type 2 diabetes: a focus on potential mechanisms. Br J Psychiatry. 2016;209(2):142-149.

36. Treadway MT, Zald DH. Reconsidering anhedonia in depression: lessons from translational neuroscience. Neurosci Biobehav Rev. 2011;35(3):537-555.

37. Fisher L, Skaff MM, Mullan JT, Arean P, Glasgow R, Masharani U. A longitudinal study of affective and anxiety disorders, depressive affect and diabetes distress in adults with type 2 diabetes. Diabet Med. 2008;25(9):1096-1101.

38. Fisher L, Gonzalez JS, Polonsky WH. The confusing tale of depression and distress in patients with diabetes: a call for greater clarity and precision. Diabet Med. 2014;31(7):764-772.

39. Katon WJ, Russo JE, Heckbert SR, et al. The relationship between changes in depression symptoms and changes in health risk behaviors in patients with diabetes. Int J Geriatr Psychiatry. 2010;25(5):466-475.

40. Fisher L, Hessler DM, Polonsky WH, Mullan J. When is diabetes distress clinically meaningful? Establishing cut points for the Diabetes Distress Scale. Diabetes Care. 2012;35(2):259-264.

41. Fritschi C, Quinn L. Fatigue in patients with diabetes: a review. J Psychosom Res. 2010;69(1):33-41.

42. Snoek FJ, Bremmer MA, Hermanns N. Constructs of depression and distress in diabetes: time for an appraisal. Lancet Diabetes Endocrinol. 2015;3(6):450-460.

43. Ismail K, Moulton CD, Winkley K, et al. The association of depressive symptoms and diabetes distress with glycaemic control and diabetes complications over 2 years in newly diagnosed type 2 diabetes: a prospective cohort study. Diabetologia. 2017;60(10):2092-2102.

44. Sun JC, Xu M, Lu JL, et al. Associations of depression with impaired glucose regulation, newly diagnosed diabetes and previously diagnosed diabetes in Chinese adults. Diabet Med. 2015;32(7):935-943.

45. Fisher L, Mullan JT, Arean P, Glasgow RE, Hessler D, Masharani U. Diabetes distress but not clinical depression or depressive symptoms is associated with glycemic control in both cross-sectional and longitudinal analyses. Diabetes Care. 2010;33(1):23-28.

46. Carter J, Cogo-Moreira H, Herrmann N, et al. Validity of the center for epidemiological studies Depression Scale in type 2 diabetes. J Psychosom Res. 2016;90:91-97.

47. Park H, Park C, Quinn L, Fritschi C. Glucose control and fatigue in type 2 diabetes: the mediating roles of diabetes symptoms and distress. J Adv Nurs. 2015;71(7):1650-1660.

48. Swardfager W, Rosenblat JD, Benlamri M, McIntyre RS. Mapping inflammation onto mood: inflammatory mediators of anhedonia. Neurosci Biobehav Rev. 2016;64:148-166.

49. Drivsholm T, de Fine ON, Nielsen AB, Siersma V. Symptoms, signs and complications in newly diagnosed type 2 diabetic patients, and their relationship to glycaemia, blood pressure and weight. Diabetologia. 2005;48(2):210-214.

50. Marin RS, Biedrzycki RC, Firinciogullari S. Reliability and validity of the apathy evaluation scale. Psychiatry Res. 1991;38(2):143-162.

51. Bruce DG, Nelson ME, Mace JL, Davis WA, Davis TME, Starkstein SE. Apathy in older patients with type 2 diabetes. Am J Geriatr Psychiatry. 2015;23(6):615-621.

52. Lunghi C, Moisan J, Gregoire JP, Guenette L. Incidence of depression and associated factors in patients with type 2 diabetes in Quebec, Canada: a population-based cohort study. Medicine. 2016;95(21):e3514. 
53. Sajatovic M, Gunzler D, Einstadter D, et al. Clinical characteristics of individuals with serious mental illness and type 2 diabetes. Psychiatr Serv. 2015;66(2):197-199.

54. Sajatovic M, Gunzler D, Einstadter D, et al. A preliminary analysis of individuals with serious mental illness and comorbid diabetes. Arch Psychiatr Nurs. 2016;30(2):226-229.

55. Cebul RD, Love TE, Jain AK, Hebert CJ. Electronic health records and quality of diabetes care. $N$ Engl J Med. 2011;365:825-833.

56. Lavretsky H, Zheng L, Weiner MW, et al. The MRI brain correlates of depressed mood, anhedonia, apathy, and anergia in older adults with and without cognitive impairment or dementia. Int J Geriatr Psychiatry. 2008;23(10):1040-1050.

57. Deschênes SS, Burns RJ, Pouwer F, Schmitz N. Diabetes complications and depressive symptoms: prospective results from the montreal diabetes health and well-being study. Psychosom Med. 2017;79(5): 603-612.

58. Kasteleyn MJ, de Vries L, van Puffelen AL, et al. Diabetes-related distress over the course of illness: results from the diacourse study Diabet Med. 2015;32(12):1617-1624.

59. Leigh Gibson E. Emotional influences on food choice: sensory, physiological and psychological pathways. Physiol Behav. 2006;89(1):53-61.

60. Leventhal AM. Relations between anhedonia and physical activity. Am J Health Behav. 2012;36(6):860-872.

61. Holmes-Truscott E, Skinner TC, Pouwer F, Speight J. Explaining psychological insulin resistance in adults with non-insulin-treated type 2 diabetes: the roles of diabetes distress and current medication concerns. Results from diabetes MILES - Australia. Prim Care Diabetes. 2016;10(1):75-82.

62. Almeida OP, Hankey GJ, Yeap BB, Golledge J, Norman PE, Flicker L. Depression, frailty, and all-cause mortality: a cohort study of men older than 75 years. J Am Med Dir Assoc. 2015;16(4):296-300.

63. Morley JE. Diabetes, sarcopenia, and frailty. Clin Geriatr Med. 2008;24(3):455-469.

64. Tanenbaum ML, Ritholz MD, Binko DH, Baek RN, Erica Shreck MS, Gonzalez JS. Probing for depression and finding diabetes: a mixedmethods analysis of depression interviews with adults treated for type 2 diabetes. J Affect Disord. 2013;150(2):533-539.

65. Lobbestael J, Leurgans M, Arntz A. Inter-rater reliability of the structured clinical interview for DSM-IV axis I disorders (SCID I) and axis II disorders (SCID II). Clin Psychol Psychother. 2011;18(1):75-79.

66. Thombs BD, Rice DB. Sample sizes and precision of estimates of sensitivity and specificity from primary studies on the diagnostic accuracy of depression screening tools: a survey of recently published studies. Int J Methods Psychiatr Res. 2016;25(2):145-152.

67. van Dijk SEM, Adriaanse MC, van der Zwaan L, et al. Measurement properties of depression questionnaires in patients with diabetes: a systematic review. Qual Life Res. 2018;27(6):1415-1430.

68. Teresi JA, Ramirez M, Lai J-S, Silver S. Occurrences and sources of Differential Item Functioning (DIF) in patient-reported outcome measures: description of DIF methods, and review of measures of depression, quality of life and general health. Psychol Sci $Q$. 2008;50(4):538.

69. Gunzler DD, Morris N. A tutorial on structural equation modeling for analysis of overlapping symptoms in co-occurring conditions using MPlus. Stat Med. 2015;34(24):3246-3280.

70. Lustman PJ, Clouse RE, Griffith LS, Carney RM, Freedland KE. Screening for depression in diabetes using the beck depression inventory. Psychosom Med. 1997;59(1):24-31.

71. Bech P, Gudex C, Staehr Johansen K. The who (ten) well-being index validation in diabetes. Psychother Psychosom. 1996;65(4):183-190.

72. Van Steenbergen-Weijenburg KM, De Vroege L, Ploeger RR, et al. Validation of the PHQ-9 as a screening instrument for depression in diabetes patients in specialized outpatient clinics. BMC Health Serv Res. 2010;10:235.

73. Swardfager W, Herrmann N, Marzolini S, et al. Major depressive disorder predicts completion, adherence, and outcomes in cardiac rehabilitation: a prospective cohort study of 195 patients with coronary artery disease. J Clin Psychiatry. 2011;72(9):1181-1188.
74. Carleton RN, Thibodeau MA, Teale MJN, et al. The center for epidemiologic studies Depression Scale: a review with a theoretical and empirical examination of item content and factor structure. PLoS One. 2013;8(3):e58067.

75. Hermanns N, Caputo S, Dzida G, Khunti K, Meneghini LF, Snoek F. Screening, evaluation and management of depression in people with diabetes in primary care. Prim Care Diabetes. 2013;7(1):1-10.

76. Anderson RJ, Freedland KE, Clouse RE, Lustman PJ. The prevalence of comorbid depression in adults with diabetes: a meta-analysis. Diabetes Care. 2001;24(6):1069-1078.

77. Wang YP, Gorenstein C. Psychometric properties of the Beck Depression Inventory-II: a comprehensive review. Rev Bras Psiquiatr. 2013;35(4):416-431.

78. Beck AT, Steer RA, Carbin MG. Psychometric properties of the Beck Depression Inventory: twenty-five years of evaluation. Clin Psychol Rev. 1988;8(1):77-100.

79. Wang Y-P, Gorenstein C. Assessment of depression in medical patients: a systematic review of the utility of the beck depression inventory-II Clinics. 2013;68(9):1274-1287.

80. Aujla N, Skinner TC, Khunti K, Davies MJ. The prevalence of depressive symptoms in a white European and South Asian population with impaired glucose regulation and screen-detected type 2 diabetes mellitus: a comparison of two screening tools. Diabet Med. 2010;27(8):896-905

81. Hajos TRS, Pouwer F, Skovlund SE, et al. Psychometric and screening properties of the WHO-5 well-being index in adult outpatients with type 1 or type 2 diabetes mellitus. Diabet Med. 2013;30(2):e63-e69.

82. Jahoda M. Current Concepts of Positive Mental Health. Vol. 61. 1958 New York: Basic Books.

83. Manea L, Gilbody S, Hewitt C, et al. Identifying depression with the PHQ-2: a diagnostic meta-analysis. JAffect Disord. 2016;203:382-395.

84. De Cock ESA, Emons WHM, Nefs G, Pop VJM, Pouwer F. Dimensionality and scale properties of the Edinburgh Depression Scale (EDS) in patients with type 2 diabetes mellitus: the DiaDDzoB study. $B M C$ Psychiatry. 2011;11:141.

85. Brouwers EPM, van Baar AL, Pop VJM. Does the Edinburgh Postnatal Depression Scale measure anxiety? J Psychosom Res. 2001;51(5):659-663.

86. Tuohy A, McVey C. Subscales measuring symptoms of non-specific depression, anhedonia, and anxiety in the Edinburgh Postnatal Depression Scale. Br J Clin Psychol. 2008;47(2):153-169.

87. van der Feltz-Cornelis CM, Nuyen J, Stoop C, et al. Effect of interventions for major depressive disorder and significant depressive symptoms in patients with diabetes mellitus: a systematic review and meta-analysis. Gen Hosp Psychiatry. 2010;32(4):380-395.

88. Lustman PJ, Clouse RE. Depression in diabetic patients: the relationship between mood and glycemic control. J Diabetes Complications. 2005;19(2):113-122.

89. Lustman PJ, Clouse RE, Nix BD, et al. Sertraline for prevention of depression recurrence in diabetes mellitus: a randomized, double-blind, placebo-controlled trial. Arch Gen Psychiatry. 2006;63(5):521-529.

90. Markowitz S, Gonzalez JS, Wilkinson JL, Safren SA. Treating depression in diabetes: emerging findings. Psychosomatics. 2011;52(1):1-18.

91. Anderberg RH, Richard JE, Hansson C, Nissbrandt H, Bergquist F, Skibicka KP. GLP-1 is both anxiogenic and antidepressant; divergent effects of acute and chronic GLP-1 on emotionality. Psychoneuroendocrinology. 2016;65:54-66.

92. Sharma S, Fulton S. Diet-induced obesity promotes depressive-like behaviour that is associated with neural adaptations in brain reward circuitry. Int J Obes. 2013;37(3):382-389.

93. Scranton R, Cincotta A. Bromocriptine - unique formulation of a dopamine agonist for the treatment of type 2 diabetes. Expert Opin Pharmacother. 2010;11(2):269-279.

94. Davis LM, Michaelides M, Cheskin LJ, et al. Bromocriptine administration reduces hyperphagia and adiposity and differentially affects dopamine D2 receptor and transporter binding in leptin-receptordeficient Zucker rats and rats with diet-induced obesity. Neuroendocrinology. 2009;89(2):152-162. 
95. Padala PR, Padala KP, Lensing SY, et al. Methylphenidate for apathy in community-dwelling older veterans with mild Alzheimer's disease: a double-blind, randomized, placebo-controlled trial. Am J Psychiatry. 2018;175(2):159-168.

96. Rosenberg PB, Lanctôt KL, Drye LT, et al. Safety and efficacy of methylphenidate for apathy in Alzheimer's disease: a randomized, placebo-controlled trial. J Clin Psychiatry. 2013;74(8):810-816.

97. Stuart MJ, Baune BT. Depression and type 2 diabetes: inflammatory mechanisms of a psychoneuroendocrine co-morbidity. Neurosci Biobehav Rev. 2012;36(1):658-676.

98. Laake J-PS, Stahl D, Amiel SA, et al. The association between depressive symptoms and systemic inflammation in people with type 2 diabetes: findings from the South London Diabetes Study. Diabetes Care. 2014;1:1-7.

99. Kappelmann N, Lewis G, Dantzer R, Jones P, Khandaker G. Antidepressant activity of anti-cytokine treatment: a systematic review and meta-analysis of clinical trials of chronic inflammatory conditions. Mol Psychiatry. 2018;23(2):335-343.

100. Raison CL, Rutherford RE, Woolwine BJ, et al. A randomized controlled trial of the Tumor Necrosis Factor-alpha antagonist infliximab in treatment resistant depression: role of baseline inflammatory biomarkers. JAMA Psychiatry. 2013;70(1):31-41.

101. Almeida OP, Flicker L, Yeap BB, Alfonso H, McCaul K, Hankey GJ. Aspirin decreases the risk of depression in older men with high plasma homocysteine. Transl Psychiatry. 2012;2:e151.

102. Mendlewicz J, Kriwin P, Oswald P, Souery D, Alboni S, Brunello N. Shortened onset of action of antidepressants in major depression using acetylsalicylic acid augmentation: a pilot open-label study. Int Clin Psychopharmacol. 2006;21(4):227-231.

103. Lustman PJ, Griffith LS. Cognitive behavior therapy for depression in type 2 diabetes mellitus. Ann Intern Med. 1998;129(8):613-621.

104. Bogner HR, Morales KH, de Vries HF, Cappola AR. Integrated management of type 2 diabetes mellitus and depression treatment to improve medication adherence: a randomized controlled trial. Ann Fam Med. 2012;10(1):15-22.

105. Madden KM. Evidence for the benefit of exercise therapy in patients with type 2 diabetes. Diabetes Metab Syndr Obes. 2013;6:233-239.

106. Mota-Pereira J, Silverio J, Carvalho S, Ribeiro JC, Fonte D, Ramos J. Moderate exercise improves depression parameters in treatmentresistant patients with major depressive disorder. J Psychiatr Res. 2011;45(8):1005-1011.

107. Petrak F, Herpertz S, Albus C, et al. Cognitive behavioral therapy versus sertraline in patients with depression and poorly controlled diabetes: the Diabetes and Depression (DAD) Study: a randomized controlled multicenter trial. Diabetes Care. 2015;38(5):767-775.

108. Bauer AM, Parker MM, Schillinger D, et al. Associations between antidepressant adherence and shared decision-making, patientprovider trust, and communication among adults with diabetes: diabetes study of northern California (DISTANCE). J Gen Intern Med. 2014;29(8):1139-1147.

109. Rethorst CD, Wipfli BM, Landers DM. The antidepressive effects of exercise. Sport Med. 2009;39(6):491-511.

110. Snowling NJ, Hopkins WG. Effects of different modes of exercise training on glucose control and risk factors for complications in type 2 diabetic patients: a meta-analysis. Diabetes Care. 2006;29(11):2518-2527.

111. Herrera JJ, Fedynska S, Ghasem PR, et al. Neurochemical and behavioural indices of exercise reward are independent of exercise controllability. Eur J Neurosci. 2016;43(9):1190-1202.

112. van der Heijden MM, van Dooren FE, Pop VJ, Pouwer F. Effects of exercise training on quality of life, symptoms of depression, symptoms of anxiety and emotional well-being in type 2 diabetes mellitus: a systematic review. Diabetologia. 2013;56(6):1210-1225.

113. Johnson JA, Al Sayah F, Wozniak L, et al. Controlled trial of a collaborative primary care team model for patients with diabetes and depression: rationale and design for a comprehensive evaluation. $B M C$ Health Serv Res. 2012;12:258.
114. Khamseh ME, Baradaran HR, Javanbakht A, Mirghorbani M, Yadollahi Z, Malek M. Comparison of the CES-D and PHQ-9 depression scales in people with type 2 diabetes in Tehran, Iran. BMC Psychiatry. 2011;22(11):1619-1623.

115. Georgiades A, Zucker N, Friedman KE, et al. Changes in depressive symptoms and glycemic control in diabetes mellitus. Psychosom Med. 2007;69(3):235-241.

116. van der Ven NC, Hogenelst MH, Tromp-Wever AM, et al. Short-term effects of cognitive behavioural group training (CBGT) in adult type 1 diabetes patients in prolonged poor glycaemic control. A randomized controlled trial. Diabet Med. 2005;22(11):1619-1623.

117. Lustman PJ, Williams MM, Sayuk GS, Nix BD, Clouse RE. Factors influencing glycemic control in type 2 diabetes during acute- and maintenance-phase treatment of major depressive disorder with bupropion. Diabetes Care. 2007;30(3):459-466.

118. Moskowitz JT, Epel ES, Acree M. Positive affect uniquely predicts lower risk of mortality in people with diabetes. Health Psychol. 2008;27(1S):S73-S82.

119. Tsenkova VK, Love GD, Singer BH, Ryff CD. Coping and positive affect predict longitudinal change in glycosylated hemoglobin. Health Psychol. 2008;27(2S):S163-S171.

120. Cummings DM, Kirian K, Howard G, et al. Consequences of comorbidity of elevated stress and/or depressive symptoms and incident cardiovascular outcomes in diabetes: results from the REasons for Geographic And Racial Differences in Stroke (REGARDS) study. Diabetes Care. 2016;39(1):101-109.

121. Katon WJ, Rutter C, Simon G, et al. The association of comorbid depression with mortality in patients with type 2 diabetes. Diabetes Care. 2005;28(11):2668-2672.

122. Lin EHB, Heckbert SR, Rutter CM, et al. Depression and increased mortality in diabetes: unexpected causes of death. Ann Fam Med. 2009;7(5):414-421.

123. Park M, Katon WJ, Wolf FM. Depression and risk of mortality in individuals with diabetes: a meta-analysis and systematic review. Gen Hosp Psychiatry. 2013;35(3):217-225.

124. Moran C, Phan TG, Chen J, et al. Brain atrophy in type 2 diabetes: regional distribution and influence on cognition. Diabetes Care. 2013;36(12):4036-4042.

125. Novak V, Last D, Alsop DC, et al. Cerebral blood flow velocity and periventricular white matter hyperintensities in type 2 diabetes. Diabetes Care. 2006;29(7):1529-1534.

126. Tiemeier H, Bakker SLM, Hofman A, Koudstaal PJ, Breteler MMB. Cerebral haemodynamics and depression in the elderly. J Neurol Neurosurg Psychiatry. 2002;73(1):34-39.

127. Katon WJ, Lin EHB, Williams LH, et al. Comorbid depression is associated with an increased risk of dementia diagnosis in patients with diabetes: a prospective cohort study. J Gen Intern Med. 2010;25(5):423-429.

128. Swardfager W, MacIntosh BJ. Depression, type 2 diabetes, and poststroke cognitive impairment. Neurorehabil Neural Repair. 2017;31(1):48-55.

129. Raison CL, Miller AH. When not enough is too much: the role of insufficient glucocorticoid signaling in the pathophysiology of stress-related disorders. Am J Psychiatry. 2003;160(9):1554-1565.

130. Pruzin JJ, Schneider JA, Capuano AW, et al. Diabetes, hemoglobin A1C, and regional Alzheimer disease and infarct pathology. Alzheimer Dis Assoc Disord. 2017;31(1):41-47.

131. Katon W, Von Korff M, Ciechanowski P, et al. Behavioral and clinical factors associated with depression among individuals with diabetes. Diabetes Care. 2004;27(4):914-920.

132. Schmitz N, Deschênes SS, Burns RJ, et al. Cardiometabolic dysregulation and cognitive decline: potential role of depressive symptoms. $\mathrm{Br}$ J Psychiatry. 2018;212(2):96-102.

133. Polonsky WH, Fisher L, Earles J, et al. Assessing psychosocial distress in diabetes: development of the diabetes distress scale. Diabetes Care. 2005;28:626-631. 
Diabetes, Metabolic Syndrome and Obesity: Targets and Therapy

Diabetes, Metabolic Syndrome and Obesity: Targets and Therapy is an international, peer-reviewed open-access journal committed to the rapid publication of the latest laboratory and clinical findings in the fields of diabetes, metabolic syndrome and obesity research Original research, review, case reports, hypothesis formation, expert opinion and commentaries are all considered for publication. The manuscript management system is completely online and includes a very quick and fair peer-review system, which is all easy to use. Visit http://www.dovepress.com/testimonials.php to read real quotes from published authors.

Submit your manuscript here: https://www.dovepress.com/diabetes-metabolic-syndrome-and-obesity-targets-and-therapy-journal 